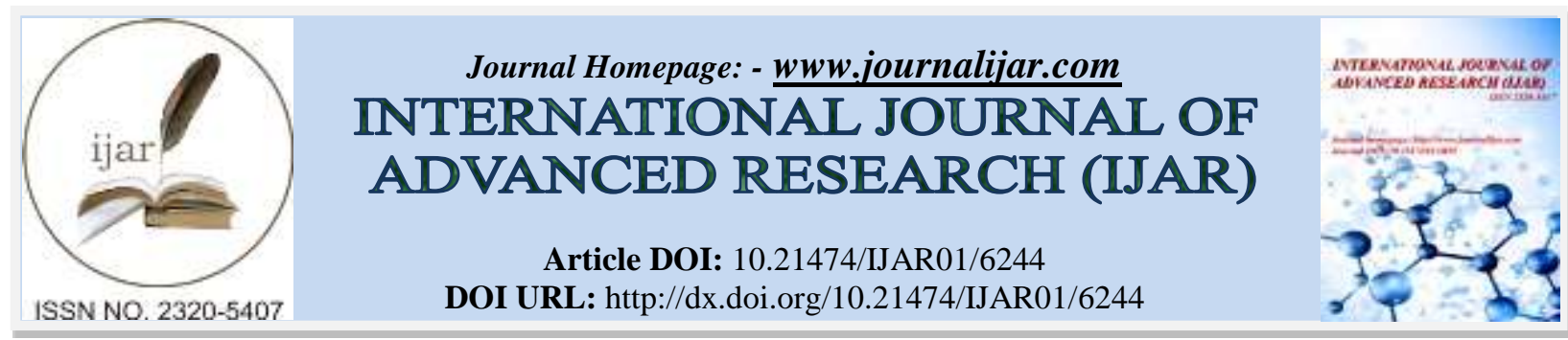

RESEARCH ARTICLE

\title{
STUDY OF ANTIBACTERIAL EFFECT OF TRIBULUS TERRESTRIS EXTRACT.
}

Mohanad H. Hussein.

Department of Medical Biotechnology, College of Biotechnology, Al-Nahrain University, Baghdad.

\section{Manuscript Info}

Manuscript History

Received: 07 November 2017

Final Accepted: 09 December 2017

Published: January 2018

Key words:-

Tribulus terrestris, antibacterial.

\begin{abstract}
This study was designed to evaluated antimicrobial activity of Tribulus terrestris aqueous extract against some pathogenic microorganisms. So that, the aqueous extract of Tribulus terrestris was screened for its antimicrobial activity using the plate agar diffusion method. It was tested against four bacteria species; two Gram-positive bacteria (Bacillus subtilis, and Staphylococcus aureus) and two Gram-negative bacteria (Escherichia coli and Pseudomonas aeruginosa). The susceptibility of these different bacterial species toward the extracts of this plant was compared with each other and with selected antibiotic (streptomycin) used as positive control. Result showed that aqueous extract of Tribulus terrestris had antibacterial
\end{abstract}

Copy Right, IJAR, 2018,. All rights reserved.

\section{Introduction:-}

The genus Tribulus terrestris (Zygophyllaceae) comprises with 20 species which grow as shrubs in subtropical areas around the world and only two species distributed in China, T. terrestris and T. cistoides. In traditional Chinese medicine, the fruit of $T$. terrestris, which is known as " $\mathrm{Ci} \mathrm{Ji} \mathrm{Li}$ ", has been used against diverse diseases for a long time. The crude saponin fraction of the whole plant has been used as a convivial drug Tribulus terrestris (L.,) is also known as puncture vine or small caltrops 10 to $60 \mathrm{~cm}$ height, annual herb, with pinnate leaves and yellow flowers . Its carpel fruits are very distinguishing in nature and are known as "Chih-hsing" in China or "Goat head" in the USA. The plant can be found in arid climate regions around the world as in southern USA, Mexico, Spain , Bulgaria, India, and China (Mahammad et al., 2012).

Taxonomy of the Plant

Class Dicotyledons

Sub class Polypetatae

Series Thalamiflorae

Order Geraniales

Family Zygophyllaceae

Genus Tribulus

Species terrestris8

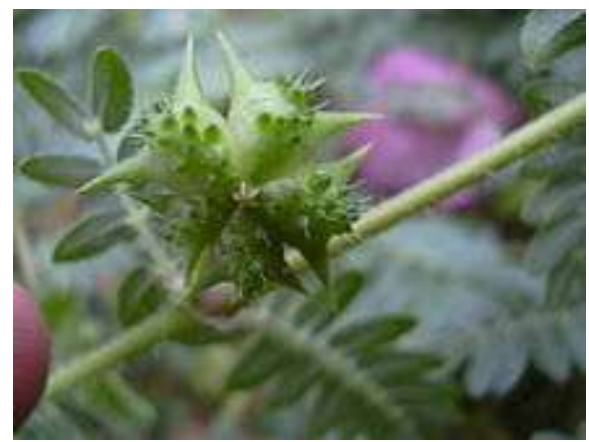

Materials and Methods:-

Plant material: Purchase from local market.

Method of extraction: A quantity of $50 \mathrm{~g}$ of the flower powder was mixed with $250 \mathrm{ml}$ sterile double distilled water. The mixture was left in a shaker incubator at $50^{\circ} \mathrm{C}$ for 24 hours, than filtered through a filter paper 
(Whatman No. 1). The filtrate was concentrated using rotary evaporator at $40^{\circ} \mathrm{C}$ until dryness and the extract residue was weighted and kept until use (Zheng et al., 1990).

Phytochemical evaluation: Phytochemical examinations were carried out for all the extracts as per the standard methods (Su et al., 2012). Detection of alkaloids, flavonoids, saponins, tannins, trepans and steroids.

Preparation of Tested Microorganisms: The average number of viable Bacillus subtilis, Staphylococcus aureus, Escherichia coli, and Pseudomonas aeruginosa microorganisms per $\mathrm{ml}$ of the stock suspensions was determined by means of the surface viable counting technique (Chakraborthy et al., 2011). About $\left(10^{8}-10^{9}\right)$ colony-forming units per $\mathrm{ml}$ was used. Each time, a fresh stock suspension was prepared; the experimental conditions were maintained constant so that bacterial suspensions with closed viable counts would be obtained.

In Vitro Testing Anti-Microbial Activity of extract: The cup-plate agar diffusion method was adopted according to the method described elsewhere in order to assess the antibacterial activity of the prepared extracts. An amount of $0.6 \mathrm{ml}$ of standardized bacterial stock suspensions $\left(10^{8}-10^{9}\right)$ colony- forming units per ml was aliquot of mixed with $60 \mathrm{ml}$ of sterile nutrient agar. About $20 \mathrm{ml}$ of the inoculated nutrient agar were distributed into sterile Petri dishes. The agar was left to set in each of these plates. Bores were made on the medium using sterile borer and 0.1 $\mathrm{ml}$ of the extracts were added to bore at conc. $20 \mathrm{mg} / \mathrm{ml}$ and $0.1 \mathrm{ml}$ of the standard Streptomycin at a concentration of $100 \mu \mathrm{g} / \mathrm{ml}$ was taken as standard The plates were then incubated in the upright position at $37^{\circ} \mathrm{C}$ for $18 \mathrm{hrs}$. Two replicates were carried out for the extract against each of the tested organism. After incubation, the diameters of growth inhibition zones were measured (Toshiyuki et al., 2001).

\section{Results:-}

Phytochemical investigation of aqueous extracts of Tribulus terrestris. Tribulus terrestris exhibited presence of saponins, terpenes, tannins and flavanoids in its extract. (Table 1).

Table 1:- Aqueous active compounds in Tribulus terrestris extract

\begin{tabular}{|ll|l|c|}
\hline No. & Active compounds & Aqueous extract of $\boldsymbol{T}$. terrestris \\
\hline 1. 1: & Tannins & + \\
\hline 2. 2: & Saponins & + \\
\hline 3. 6: & Alkaloids & + \\
\hline 4. 7: & Flavonoids & + \\
\hline 5. 9: & Terpenes & Steroid & + \\
\hline
\end{tabular}

The symbol (+) refers to presence of compound and the symbol (-) refers to absence of cmpound.

Antibacterial activity of Tribulus terrestris extract against the tested organisms are as follows: In case of Tribulus terrestris, aqueous extracts inhibited the growth of the bacteria used in the study. (Table.2) and (figure.1)

Table 2:- Antibacterial activity of Tribulus terrestris against some pathogenic bacterial species as compared with streptomycin.

\begin{tabular}{|l|c|c|c|c|}
\hline \multirow{2}{*}{ Drug } & \multicolumn{3}{|c|}{ Mean Diameter of Growth Inhibition Zone in (mm). } \\
\cline { 2 - 5 } & B. subtilis & S. aureus & E. coli & P. aeruginosa \\
\hline Tribulus terrestris & 17 & 15 & 0 & 0 \\
\hline streptomycin & 35 & 25 & 20 & 0 \\
\hline
\end{tabular}

\section{Discussion:-}

Tribulus contains biologically active substances as steroids, saponins, flavonoids, alkaloids. Glycoconjugates are a class of complex molecules that are widely distributed in the plant kingdom and in some marine organisms. This class of compounds has a wide range of biological activates as anti-inflammatory, antimicrobial, antifungal, anticancer and other benefits. Among these compounds, steroidal and triterpenoid saponins have long been known as components of widely used herbal drugs and pharmaceutical preparation (Javed et al., 2012). 


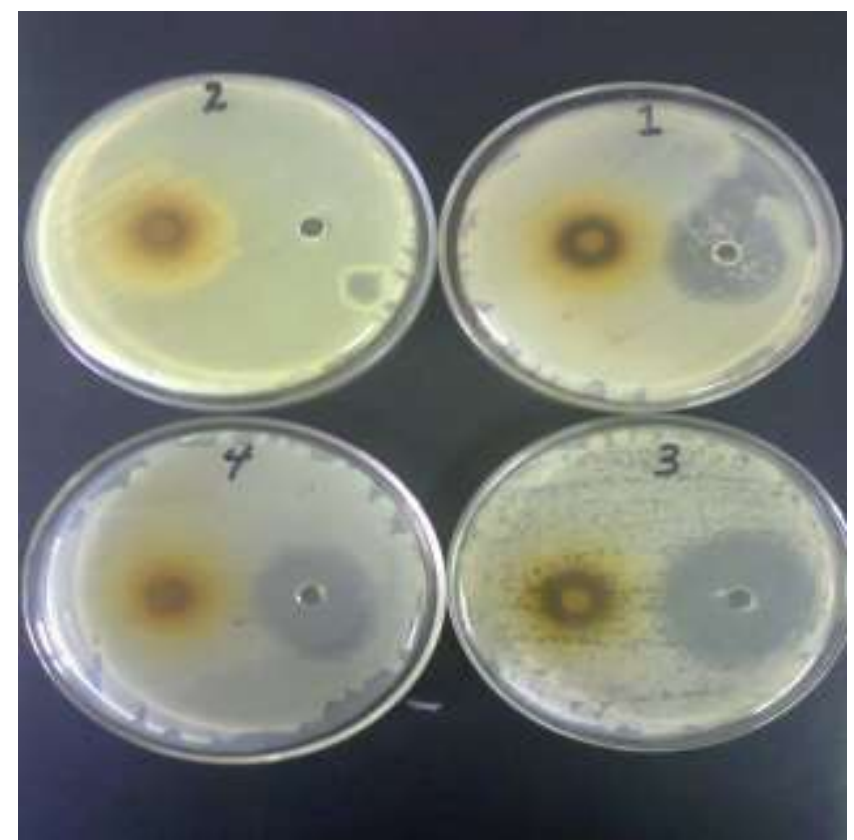

Figure.1:- Antibacterial activity of of Tribulus terrestris extract against compared with streptomycin (1) Staphylococcus aureus, (2) Pseudomonas aeruginosa, (3) Bacillus subtilis, and (4) E. coli.

The saponins are distinguished as steroidal saponins and triterpenoid. The steroidal saponins include the following chemicals: terrestrosin, diosin, gracillin, kikuba, PTN, nohecogenin, glucoside, tribulosi and Fgitonis (Lee.,2012).The other constituents are flavonoids which include the following substance: Kaempferol, kaempferol glycosides and quercertin. The other minor constituents are present like fatty acids (palmitic, stearic, oleic and linoleic) acids (Kaul et al., 2012).

Tribulus also contains small amounts of alkaloid, tannin, potassium salts, cinnamic amide, resine and sugar. It also presents fixed and essential oils, porphyrin, saprogenic ,25 species of flavonoids-glycosides and resin . Isolation of four furostanol saponins are known as: methylprotodioscin, protodioscin, methylprototribestin and prototribestin. The quality of this herb is dependent on the PTN content (Mona et al., 2014).

\section{References:-}

1. Chakraborthy,G.S.; Arora, R.; and Majee, C. (2011). International research journal of pharmacy., 2(1): 61-65.

2. Javed Akhtar Ansaria ; Mateen Sayyeda and Qamruzzamab, (2012). Analgesic and Anti-Inflammatory Effect of Ethanol Extract of Tribulus Terrestris L. Research Journal of Pharmaceutical, Biological and Chemical Sciences . $3(4): 1365$

3. Ka ul, N.; Ag r a wa l, H.; Pa ti l, B.; Ka kad, A. and Dha R. (2005). Application on of stability - indicating HPTLC method for quantitative determination of metadoxine in pharmaceutical dosage form. Farmaco $60: 351-360$.

4. Mahammad ,S.; Maruthi,E.; Bheemewsaraiah,K.; S. Manjunatha,S. and Kodidhela, L. (2012) Effect of Tribulus terrestris (L.) on liver in Isoproterenol-Induced Myocardial Infarction. International Journal of Research in Biochemistry and Biophysics . 2(4): 10-12

5. Mona,S.; Mohamed, F. and Wadah, J. (2014). Chromatographic finger print analysis of anti-inflammatory active extract fractions of aerial parts of Tribulus terrestris by HPTLC technique Asian Pac J Trop Biomed. 4(3): 203-208

6. Su, S.; Hua, Y.; Wang, Y. ; Gu, W.; Zhou, W. ; Duan, JA.; Jiang, H. ; Chen, T. and Tang, Y.(2012). J Ethnopharmacol 139: 649-56.

7. Toshiyuki Marukami; Akinobu Kishi; and Masayuki Yoshikawa (2001). Chem. Pharm. Bull, 49(8): 974-978.

8. Zheng, M.; Sakai,I.; Ose,Y.; and Nakane,H. (1990).Antimutagenic activity by medical plants in trailonal Chinese medicines. Shoy. Akugaku.,; 44(3): 225-229. 